\title{
Robust Ranking and Selection with Optimal Computing Budget Allocation *
}

\author{
Siyang Gao ${ }^{\mathrm{a}, 1}$, Hui Xiao $^{\mathrm{b}}$, Enlu Zhou ${ }^{\mathrm{c}}$, Weiwei Chen ${ }^{\mathrm{d}}$ \\ ${ }^{a}$ Department of Systems Engineering and Engineering Management, City University of Hong Kong, Tat Chee Avenue, \\ Kowloon, Hong Kong \\ ${ }^{\mathrm{b}}$ School of Statistics, Southwestern University of Finance and Economics, Chengdu 611130, China \\ ${ }^{\mathrm{c}}$ H. Milton Stewart School of Industrial and Systems Engineering, Georgia Institute of Technology, GA 30332, USA \\ ${ }^{\mathrm{d}}$ Department of Supply Chain Management, Rutgers University, 1 Washington Park, Newark, NJ 07102, USA
}

\begin{abstract}
In this paper, we consider the ranking and selection $(\mathrm{R} \& S)$ problem with input uncertainty. It seeks to maximize the probability of correct selection (PCS) for the best design under a fixed simulation budget, where each design is measured by their worst-case performance. To simplify the complexity of PCS, we develop an approximated probability measure and derive an asymptotically optimal solution of the resulting problem. An efficient selection procedure is then designed within the optimal computing budget allocation (OCBA) framework. More importantly, we provide some useful insights on characterizing an efficient robust selection rule and how it can be achieved by adjusting the simulation budgets allocated to each scenario.
\end{abstract}

Key words: Simulation optimization; ranking and selection; OCBA; robust optimization; computing budget allocation.

\section{Introduction}

Many problems in control engineering involve large-scale discrete-event dynamic systems. Since these systems are usually too complex to be described by succinct mathematical models, stochastic simulation has been a popular choice for analyzing these systems. However, due to slow convergence, simulation efficiency is a major concern, especially when the number of competing designs to be compared is large. This explains the increasing popularity of research in ranking and selection (R\&S): techniques that determine the number of simulation replica-

\footnotetext{
* This work has been supported in part by Hong Kong Research Grants Council (Grant 21204316), National Science Foundation of China (Grants 61603321 and 71601158) and City University of Hong Kong (Grants 7200419 and 7004464 ). The material in this paper was partially presented at the 2016 Winter Simulation Conference, December 11-14, 2016, Arlington, VA, USA.

Email addresses: siyangao@cityu.edu.hk (Siyang Gao), msxh@swufe.edu.cn (Hui Xiao)

enlu.zhou@isye.gatech.edu (Enlu Zhou), wchen@business.rutgers.edu (Weiwei Chen).

1 Corresponding author. Tel.: +852 3442 4759; fax: +852 34420173.
}

tions for each design alternative such that the selection quality for the best design is optimized or guaranteed at a pre-specified level.

There are two primary approaches for R\&S problems: indifference-zone (IZ) and optimal computing budget allocation (OCBA). The IZ approach aims to provide a guarantee for the selection quality, assuming that the mean performance of the best design is at least $\delta^{*}$ better than each alternative, where $\delta^{*}$ is the minimum difference worth detecting (Kim \& Nelson, 2001). The OCBA approach allocates the samples sequentially in order to optimize the selection quality under a simulation budget constraint (Chen et al., 2000; Gao \& Chen, 2015, 2016b). The high efficiency of the OCBA method has been demonstrated via a variety of numerical experiments (Branke et al., 2007; Chen et al., 2014).

An implicit assumption for the abovementioned R\&S procedures is that the true input distributions and their parameters are known, while in practice, they are typically unknown and have to be estimated from limited historical data. The finiteness of historical data leads to uncertainty in the estimated input distributions and their parameters, which might (severely) affect the qual- 
ity of the selection in $R \& S$ procedures.

In view of the importance of this issue, Corlu \& Biller (2013) developed a subset selection procedure that accounts for the input uncertainty. Fan et al. (2013) presented a robust IZ-based R\&S formulation that selects the best design with respect to the worst-case choices among a finite collection of possible input models, called robust selection of the best (RSB). This issue is also related to two streams of literature. The first is input uncertainty quantification, which quantifies the impact of input uncertainty on the simulation output (Barton et al., 2014; Song et al., 2014). The other is robust optimization (RO) (Delage \& Ye, 2010; Ben-Tal et al., 2013). Different from simulation-based optimization in which targeted problems do not have nice structures to be exploited, $\mathrm{RO}$ often requires the optimization problems to be available explicitly in closed form.

In this research, we utilize the OCBA approach and develop a new and efficient procedure for R\&S problems with input uncertainty. To the best of our knowledge, it is the first OCBA-based procedure for $\mathrm{R} \& \mathrm{~S}$ problems with input uncertainty. The uncertainty set is assumed to contain a finite number of scenarios for the underlying input distributions and parameters. The selection problem is formulated as maximizing the probability of correctly selecting the best design under a fixed simulation budget, where the performance of a design is measured by its worst-case performance among all the possible scenarios in the uncertainty set. When all the scenarios of each design and their means and variances are known, we derive the asymptotic (as the simulation budget goes to infinity) optimal solution for the selection problem considered. A sequential selection algorithm, called R-OCBA (robust OCBA), is designed which heuristically implements the derived solution. The significantly higher efficiency of R-OCBA is demonstrated via numerical tests. A preliminary study of this problem has been presented without proof in Gao et al. (2016).

The rest of the paper is organized as follows. Section 2 formulates the selection problem with input uncertainty. Section 3 derives the asymptotic optimal solution for the problem formulated and develops a corresponding sequential selection procedure. Numerical experiments are provided in Section 4, followed by conclusions in Section 5 .

\section{Problem Statement}

In this section, we provide some notation and assumptions and formulate the selection problem with input uncertainty.

\subsection{Preliminaries}

Essentially, we want to solve the following selection problem

$$
\min _{x \in \mathscr{X}} E_{P}[h(x, \xi)]
$$

where the set of system designs (solutions) $\mathscr{X}=$ $\left\{x_{1}, x_{2}, \ldots, x_{k}\right\}$ is non-empty and finite. The performance measure function $E_{P}[h(x, \xi)]$ has no analytical form and must be evaluated via simulation. $h(x, \xi)$ is some random estimate of the performance of the system given a design $x$. $\xi$ represents the random noise of the system and follows an unknown distribution $P$.

In practice, the functional forms and the associated parameters of distribution $P$ are estimated from historical data, which leads to uncertainty of $P$. To model this input uncertainty, we follow Fan et al. (2013) and assume that for all the designs in $\mathscr{X}$, the set of possible distributions of $\xi$ is identical and contains a finite number of elements, denoted as $\mathscr{P}=\left\{P_{1}, P_{2}, \ldots, P_{m}\right\}$. We call $\mathscr{P}$ the uncertainty set and an element in $\mathscr{P}$ a scenario. Note that $\mathscr{P}$ incorporates the uncertainty from both the input distribution and its associated parameters and $h(x, \xi)$ is random given any $P_{j} \in \mathscr{P}$. In order to obtain a finite number of scenarios, we can first identify a number of appropriate input distributions from historical data and then discretize the possible ranges of the associated parameters to establish $\mathscr{P}$.

To facilitate our presentation, we call $x_{i}$ design $i$ and $P_{j}$ scenario $j$ with $i \in\{1,2, \ldots, k\}$ and $j \in\{1,2, \ldots, m\}$. We define the following notation:

$X_{i, j, t}$ : output of the $t$-th simulation replication for scenario $j$ of design $i, i \in\{1,2, \ldots, k\}$ and $j \in\{1,2, \ldots, m\}$;

$\mu_{i, j}$ : mean of $X_{i, j, t}$, i.e., $\mu_{i, j}=E\left[X_{i, j, t}\right]$;

$\sigma_{i, j}^{2}$ : variance of $X_{i, j, t}$, i.e., $\sigma_{i, j}^{2}=\operatorname{Var}\left[X_{i, j, t}\right]$;

$n$ : total number of simulation replications (budget);

$n_{i, j}$ : number of simulation replications allocated to scenario $j$ of design $i$;

$\alpha_{i, j}$ : proportion of the total simulation budget allocated to scenario $j$ of design $i$;

$\bar{X}_{i, j}$ : sample mean of scenario $j$ of design $i$, i.e., $\bar{X}_{i, j}=$ $\frac{1}{n_{i, j}} \sum_{t=1}^{n_{i, j}} X_{i, j, t}$

$S_{i, j}^{2}$ : sample variance of scenario $j$ of design $i$, i.e., $S_{i, j}^{2}=\frac{1}{n_{i, j}-1} \sum_{t=1}^{n_{i, j}}\left(X_{i, j, t}-\bar{X}_{i, j}\right)^{2}$;

$b$ : the true best design, $b \in\{1,2, \ldots, k\}$;

$\delta_{i j, u v}=\mu_{i, j}-\mu_{u, v}$.

In this research, the performance of design $i$ is measured by its worst-case performance among the $m$ possible scenarios in $\mathscr{P}$, i.e., $\max _{j \in\{1,2, \ldots, m\}} \mu_{i, j}$, for all $i \in\{1,2, \ldots, k\}$. This is a common setting to account for input uncertainty (e.g., Ghaoui et al., 2003; Gülpınar \& Rustem, 2007). Since making decisions based on the 
worst-case scenario can prevent potential high risk, this setting is preferred by conservative decision makers. We assume that for each design $i \in\{1,2, \ldots, k\}$, there exists scenario $j_{i}$ such that $\mu_{i, j_{i}}>\mu_{i, j}$ for all $j \in\{1,2, \ldots, m\}$ and $j \neq j_{i}$, and there exists design $i \in\{1,2, \ldots, k\}$ such that $\mu_{i, j_{i}}<\mu_{i^{\prime}, j_{i^{\prime}}}$ for all $i^{\prime} \in\{1,2, \ldots, k\}$ and $i^{\prime} \neq i$. This assumption ensures that the worst-case scenario of each design and the true best design are uniquely defined. To make the derivation more tractable, we further assume that the simulation output samples for each scenario are normally distributed for all the designs and are independent from replication to replication, as well as independent across different designs and scenarios. That is, $X_{i, j, t} \sim N\left(\mu_{i, j}, \sigma_{i, j}^{2}\right)$ and $\bar{X}_{i, j} \sim N\left(\mu_{i, j}, \frac{\sigma_{i, j}^{2}}{n_{i, j}}\right)$.

\subsection{Problem Formulation}

Given a fixed simulation budget, the best design cannot be selected with certainty, and a common way to deal with this issue is to allocate the simulation budget to maximize the probability of correct selection (PCS), i.e., the probability of correctly selecting the best design. With the performance of each design measured by their worst-case performance, a correct selection occurs when the observed worst-case scenario of the true best design $b$ is better than the observed worst-case scenarios of the other designs. Then,

$$
\begin{aligned}
P C S & =P\left\{\max _{j \in\{1, \ldots, m\}} \bar{X}_{b, j}<\min _{l \in\{1, \ldots, k\}, l \neq b \in\{1, \ldots, m\}} \max _{r, m} \bar{X}_{l, r}\right\} \\
& =P\left\{\bigcap_{l=1, l \neq b}^{k} \bigcup_{r=1}^{m} \bigcap_{j=1}^{m}\left(\bar{X}_{b, j} \leq \bar{X}_{l, r}\right)\right\}
\end{aligned}
$$

The selection problem is formulated as,

$$
\begin{array}{ll}
\max _{n_{i, j}} & P C S \\
\text { s.t. } & \sum_{i=1}^{k} \sum_{j=1}^{m} n_{i, j}=n, \\
& n_{i, j} \geq 0, i=1, \ldots, k, j=1, \ldots, m .
\end{array}
$$

In this research, we ignore the minor technicalities associated with $n_{i, j}$ 's not being integer. A major difficulty for solving (3) is that the objective function PCS is computationally intractable using the expression given in (2). To evaluate PCS in a relatively fast and inexpensive way, we present an approximation for PCS using a lower bound.

Theorem 1 The PCS in (2) is bounded from below by

$$
P C S \geq 1-\sum_{l=1, l \neq b}^{k} \sum_{j=1}^{m} P\left\{\bar{X}_{b, j}>\bar{X}_{l, r_{l}}\right\}
$$

where $r_{l}=\arg \max _{r \in\{1,2, \ldots, m\}} \mu_{l, r}$ is the worst-case scenario of design $l$.

Proof. According to (2),

$$
\begin{aligned}
& P C S=P\left\{\bigcap_{l=1, l \neq b}^{k} \bigcup_{r=1}^{m} \bigcap_{j=1}^{m}\left(\bar{X}_{b, j} \leq \bar{X}_{l, r}\right)\right\} \\
& \geq 1-\sum_{l=1, l \neq b} P\left\{\bigcap_{r=1}^{m} \bigcup_{j=1}^{m}\left(\bar{X}_{b, j}>\bar{X}_{l, r}\right)\right\} \\
& \geq 1-\sum_{l=1, l \neq b}^{k} r \in\{1,2, \ldots, m\} \\
& \geq 1-\sum_{l=1, l \neq b}^{k} r \in\{1,2, \ldots, m\} \\
& \left.\geq 1-\sum_{l=1, l \neq b}^{m} \sum_{j=1}^{m} P\left\{\bar{X}_{b, j}>\bar{X}_{l, r}\right)\right\}
\end{aligned}
$$

In the proof, the relaxation in (5) and (6) is based on Bonferroni inequality. We call the lower bound of PCS on the right-hand side of (4) approximated probability of correct selection (APCS). In this research, we consider the following optimization problem instead of (3):

$$
\begin{array}{ll}
\max _{n_{i, j}} & A P C S \\
\text { s.t. } & \sum_{i=1}^{k} \sum_{j=1}^{m} n_{i, j}=n, \\
& n_{i, j} \geq 0, i=1, \ldots, k, j=1, \ldots, m .
\end{array}
$$

This formulation falls in the OCBA framework. The simulation budget $n$ is allocated to all the scenarios of each design to maximize APCS. Although it is an approximated problem compared to (3), it provides a good starting point from which the computation is much more tractable. Further development of efficient selection rules is then possible based on (8).

\section{Selection Procedure}

In this section, we derive an asymptotic optimal solution for problem (8) and propose a sequential selection procedure for implementation. 


\subsection{Asymptotic Optimal Solution}

To solve problem (8), we start with two lemmas.

Lemma 2 (Gao $\&$ Chen, 2016a) Consider $u_{i}, v_{i}, w_{j}, z_{j} \in$ $\mathbb{R}$ with $u_{i}, w_{j}>0$ and $v_{i}, z_{j}<0, i=1,2, \ldots, m_{1}$ and $j=1,2, \ldots, m_{2}$. If for $x \in \mathbb{R}, m_{1}, m_{2} \geq 1$, $\sum_{i=1}^{m_{1}} u_{i} \exp \left(v_{i} x\right)=\sum_{j=1}^{m_{2}} w_{j} \exp \left(z_{j} x\right)$ as $x \rightarrow+\infty$, then, $\max _{i \in\left\{1, \ldots, m_{1}\right\}} v_{i}=\max _{j \in\left\{1, \ldots, m_{2}\right\}} z_{j}$.

Lemma 3 Problem (8) is an asymptotic convex optimization problem.

Proof. Since $\mu_{b, j}<\mu_{l, r_{l}}, P\left\{\bar{X}_{b, j}>\bar{X}_{l, r_{l}}\right\}$ is a convex function as $n_{b, j} \rightarrow \infty$ and $n_{l, r_{l}} \rightarrow \infty$ according to Gao \& Shi (2015). That is, APCS is an asymptotic concave function. Consequently, problem (8) is an asymptotic convex optimization problem.

With the convexity, we can use the KKT conditions to solve (8).

Theorem 4 Denote $\mathcal{R}_{b j, l r}=\frac{\delta_{b j, l r}^{2}}{2\left(\sigma_{b, j}^{2} / \alpha_{b, j}+\sigma_{l, r}^{2} / \alpha_{l, r}\right)}$ for all $l \in\{1,2, \ldots, k\}$ and $l \neq b$ and $j, r \in\{1,2, \ldots, m\}$. Define design $l_{j}$ and scenario $j_{l}$ such that $\mathcal{R}_{b j, l_{j} r_{l_{j}}}=$ $\min _{l \in\{1,2, \ldots, k\}, l \neq b} \mathcal{R}_{b j, l r_{l}}$ for $j \in\{1,2, \ldots, m\}$ and $\mathcal{R}_{b j_{l}, l r_{l}}=\min _{j \in\{1,2, \ldots, m\}} \mathcal{R}_{b j, l r_{l}}$ for $l \in\{1,2, \ldots, k\}$ and $l \neq b$. Problem (8) is asymptotically optimized if

$$
\begin{aligned}
& \sum_{j=1}^{m} \frac{n_{b, j}^{2}}{\sigma_{b, j}^{2}}=\sum_{l=1, l \neq b}^{k} \frac{n_{l, r_{l}}^{2}}{\sigma_{l, r_{l}}^{2}}, \\
& \mathcal{R}_{b j, l_{j} r_{l_{j}}}=\mathcal{R}_{b j^{\prime}, l_{j^{\prime}} r_{j^{\prime}}}, \quad j, j^{\prime} \in\{1,2, \ldots, m\}, j \neq j^{\prime}, \\
& \mathcal{R}_{b j_{l}, l r_{l}}=\mathcal{R}_{b j_{l^{\prime}}, l^{\prime} r_{l^{\prime}},}, \quad l, l^{\prime} \in\{1,2, \ldots, k\}, l \neq l^{\prime} \neq b .
\end{aligned}
$$

Proof. Let $\gamma$ be the Lagrangian multiplier associated with constraint $\sum_{i=1}^{k} \sum_{j=1}^{m} n_{i, j}=n$. The KKT condi- tions of problem (8) are reported as follows:

$$
\begin{aligned}
\frac{\partial A P C S}{\partial n_{b, j}} & =\sum_{l=1, l \neq b}^{k} \frac{-1}{2 \sqrt{2 \pi}} \exp \left(-\mathcal{R}_{b j, l r_{l}} n\right) \frac{\delta_{b j, l r_{l}} \sigma_{b, j}^{2}}{\left(\frac{\sigma_{b, j}^{2}}{n_{b, j}}+\frac{\sigma_{l, r_{l}}^{2}}{n_{l, r_{l}}}\right)^{\frac{3}{2}} n_{b, j}^{2}} \\
& =\gamma, \quad j \in\{1,2, \ldots, m\}, \\
\frac{\partial A P C S}{\partial n_{l, r_{l}}} & =\sum_{j=1}^{m} \frac{-1}{2 \sqrt{2 \pi}} \exp \left(-\mathcal{R}_{b j, l r_{l}} n\right) \frac{\delta_{b j, l r_{l}} \sigma_{l, r_{l}}^{2}}{\left(\frac{\sigma_{b, j}^{2}}{n_{b, j}}+\frac{\sigma_{l, r_{l}}^{2}}{n_{l, r_{l}}}\right)^{\frac{3}{2}} n_{l, r_{l}}^{2}} \\
& =\gamma, \quad l \in\{1,2, \ldots, k\}, l \neq b, \\
& \sum_{i=1}^{k} \sum_{j=1}^{m} n_{i, j}=n .
\end{aligned}
$$

From (12) and (13),

$$
\begin{aligned}
& \sum_{l=1, l \neq b}^{k} \sum_{j=1}^{m} \frac{-1}{2 \sqrt{2 \pi}} \exp \left(-\mathcal{R}_{b j, l r_{l}} n\right) \frac{\delta_{b j, l r_{l}}}{\left(\frac{\sigma_{b, j}^{2}}{n_{b, j}}+\frac{\sigma_{l, r_{l}}^{2}}{n_{l, r_{l}}}\right)^{\frac{3}{2}}} \\
= & \sum_{j=1}^{m} \gamma \frac{n_{b, j}^{2}}{\sigma_{b, j}^{2}}=\sum_{l=1, l \neq b}^{k} \gamma \frac{n_{l, r_{l}}^{2}}{\sigma_{l, r_{l}}^{2}} .
\end{aligned}
$$

Consequently, $\sum_{j=1}^{m} \frac{n_{b, j}^{2}}{\sigma_{b, j}^{2}}=\sum_{l=1, l \neq b}^{k} \frac{n_{l, r_{l}}^{2}}{\sigma_{l, r_{l}}^{2}}$.

Apply (12) to $j, j^{\prime} \in\{1,2, \ldots, m\}$ and $j \neq j^{\prime}$,

$$
\begin{aligned}
& \sum_{l=1, l \neq b}^{k} \frac{-1}{2 \sqrt{2 \pi}} \exp \left(-\mathcal{R}_{b j, l r_{l}} n\right) \frac{\delta_{b j, l r_{l}} \sigma_{b, j}^{2}}{\left(\frac{\sigma_{b, j}^{2}}{\alpha_{b, j}}+\frac{\sigma_{l, r_{l}}^{2}}{\alpha_{l, r_{l}}}\right)^{\frac{3}{2}} \alpha_{b, j}^{2}} \\
= & \sum_{l=1, l \neq b}^{k} \frac{-1}{2 \sqrt{2 \pi}} \exp \left(-\mathcal{R}_{b j^{\prime}, l r_{l}} n\right) \frac{\delta_{b j^{\prime}, l r_{l}} \sigma_{b, j^{\prime}}^{2}}{\left(\frac{\sigma_{b, j^{\prime}}^{2}}{\alpha_{b, j^{\prime}}}+\frac{\sigma_{l, r_{l}}^{2}}{\alpha_{l, r_{l}}}\right)^{\frac{3}{2}} \alpha_{b, j^{\prime}}^{2}} .
\end{aligned}
$$

According to Lemma 3 , as the simulation budget $n \rightarrow$ $\infty, \min _{l \in\{1,2, \ldots, k\}, l \neq b} \mathcal{R}_{b j, l r_{l}}=\min _{l \in\{1,2, \ldots, k\}, l \neq b} \mathcal{R}_{b j^{\prime}, l r_{l}}$. That is, $\mathcal{R}_{b j, l_{j} r_{l_{j}}}=\mathcal{R}_{b j^{\prime}, l_{j^{\prime}} r_{l_{j^{\prime}}}}$ for $j, j^{\prime} \in\{1,2, \ldots, m\}$ and $j \neq j^{\prime}$.

Similarly, we can apply (13) to $l, l^{\prime} \in\{1,2, \ldots, k\}$ and $l \neq l^{\prime} \neq b$. As the simulation budget $n \rightarrow \infty$, $\mathcal{R}_{b j_{l}, l r_{l}}=\mathcal{R}_{b j_{l^{\prime}}, l^{\prime} r_{l^{\prime}}}, \quad l, l^{\prime} \in\{1,2, \ldots, k\}$ for $l \neq l^{\prime} \neq b$.

Note that equations (9)-(11) correspond to the KKT conditions of the limiting problem of (8), i.e., the problem of maximizing $\lim _{n \rightarrow \infty} A P C S$. It suggests that if the solutions satisfying (9)-(11) are implemented, the optimal allocation will be achieved asymptotically. Theorem 4 provides useful insights for determining efficient selection rules for problem (8), which are summarized as follows. 
We do not need to correctly compare all the scenarios under consideration. In fact, for a design, as long as all the other $k-1$ designs have at least one scenario inferior to all the $m$ scenarios of this design, it is sufficient to conclude that this design is the best design. Following this idea, Theorem 4 suggests concentrating the simulation budget only on scenarios in $\mathscr{S}_{1}$ and $\mathscr{S}_{2}$, where $\mathscr{S}_{1}=\{$ scenario $j$ of design $b: j \in\{1,2, \ldots, m\}\}$ and $\mathscr{S}_{2}=\left\{\right.$ scenario $r_{l}$ of design $l: l \in\{1,2, \ldots, k\}$ and $l \neq$ $b\}$. That is, we only seek to correctly compare the $m$ scenarios of the best design and the worst-case scenario of all the $k-1$ non-best designs.

In order to efficiently compare the scenarios in $\mathscr{S}_{1}$ and $\mathscr{S}_{2}$, we follow optimality conditions (9), (10) and (11). Condition (9) indicates the number of simulation replications that should be spent in $\mathscr{S}_{1}$ and $\mathscr{S}_{2}$. It provides a general balance of the simulation effort devoted to the scenarios in the two sets.

To interpret condition (10), note that $\mathcal{R}_{b j, l r_{l}}$ is the rate function of probability $P\left\{\bar{X}_{b, j}>\bar{X}_{l, r_{l}}\right\}$ (Glynn \& Juneja, 2004). As the simulation budget $n \rightarrow \infty, \mathcal{R}_{b j, l r_{l}}$ describes the exponential decaying rate in which the probability of a wrong pair-wise comparison $\bar{X}_{b, j}>\bar{X}_{l, r_{l}}$ between scenario $j$ of design $b$ and scenario $r_{l}$ of design $l$ goes to 0 . The smaller $\mathcal{R}_{b j, l r_{l}}$ is, the less the decaying rate is, and the more likely we make a wrong pair-wise comparison $\bar{X}_{b, j}>\bar{X}_{l, r_{l}}$. For scenario $j$ of design $b$, de$\operatorname{sign} l_{j}$ is defined as arg $\min _{l \in\{1,2, \ldots, k\}, l \neq b} \mathcal{R}_{b j, l r_{l}}$. That is, scenario $r_{l_{j}}$ of design $l_{j}$ is the scenario in $\mathscr{S}_{2}$ that is most likely to lead to a wrong comparison with scenario $j$ of design $b . \mathcal{R}_{b j, l_{j} r_{l_{j}}}$ therefore represents the difficulty of correctly observing scenario $j$ of design $b$, i.e., correctly comparing scenario $j$ of design $b$ with all the scenarios in $\mathscr{S}_{2}$. This is because if scenario $r_{l_{j}}$ of design $l_{j}$ is correctly compared with scenario $j$ of design $b$, all the other scenarios in $\mathscr{S}_{2}$ are likely to be correctly compared with scenario $j$ of design $b$, and scenario $j$ of design $b$ is then correctly observed. By forcing $\mathcal{R}_{b j, l_{j} r_{l_{j}}}=\mathcal{R}_{b j^{\prime}, l_{j^{\prime}} r_{l_{j^{\prime}}}}$ for any two scenarios $j, j^{\prime} \in\{1,2, \ldots, m\}$ and $j \neq j^{\prime}$, condition (10) is essentially adjusting the number of simulation replications allocated to all the scenarios of design $b$ (i.e., scenarios in $\mathscr{S}_{1}$ ) such that the difficulty of correctly observing these scenarios is equal to each other.

We can perform similar analysis for condition (11) and conclude that this condition adjusts the number of simulation replications allocated to the worst-case scenario of all the non-best designs (i.e., scenarios in $\mathscr{S}_{2}$ ) such that the difficulty of correctly observing these scenarios is equal to each other.

In summary, condition (9) maintains a general balance for the simulation budget allocated to $\mathscr{S}_{1}$ and $\mathscr{S}_{2}$. Conditions (10) and (11) aim to keep appropriate proportions of the simulation budget for each scenario in $\mathscr{S}_{1}$ and $\mathscr{S}_{2}$ respectively.

\subsection{Sequential Selection Procedure}

Based on the discussion above, we develop a sequential selection procedure which heuristically implements optimality conditions (9), (10) and (11). At each iteration, we provide a small incremental budget $\Delta$ and calculate $\mathcal{A}_{1}=\sum_{j=1}^{m} \frac{n_{b, j}^{2}}{\sigma_{b, j}^{2}}$ and $\mathcal{A}_{2}=\sum_{l=1, l \neq b}^{k} \frac{n_{l, r_{l}}^{2}}{\sigma_{l, r_{l}}^{2}}$. If $\mathcal{A}_{1}<\mathcal{A}_{2}$, we allocate $\Delta$ to some scenario in $\mathscr{S}_{1}$, i.e., some scenario of the best design $b$. Otherwise, we allocate $\Delta$ to some scenario in $\mathscr{S}_{2}$, i.e., the worst-case scenario of some nonbest design. By doing so, (9) can be balanced.

To decide which scenario in $\mathscr{S}_{1}$ or $\mathscr{S}_{2}$ should receive $\Delta$, note that,

$$
\begin{gathered}
\frac{\partial \mathcal{R}_{b j, l_{j} r_{l_{j}}}}{\partial \alpha_{b, j}}=\frac{\delta_{b j, l_{j} r_{l_{j}}}^{2}}{2\left(\frac{\sigma_{b, j}^{2}}{\alpha_{b, j}}+\frac{\sigma_{l_{j}, r_{l}}}{\alpha_{l_{j}, r_{l}}}\right)^{2}} \frac{\sigma_{b, j}^{2}}{\alpha_{b, j}^{2}}>0, \\
\frac{\partial \mathcal{R}_{b j_{l}, l r_{l}}}{\partial \alpha_{l r_{l}}}=\frac{\delta_{b j_{l}, l r_{l}}^{2}}{2\left(\frac{\sigma_{b, j_{l}}^{2}}{\alpha_{b, j_{l}}}+\frac{\sigma_{l, r_{l}}^{2}}{\alpha_{l, r_{l}}}\right)^{2}} \frac{\sigma_{l, r_{l}}^{2}}{\alpha_{l, r_{l}}^{2}}>0 .
\end{gathered}
$$

Then, if $\mathcal{A}_{1}<\mathcal{A}_{2}$, we allocate the incremental budget $\Delta$ to scenario $j_{\min }$ of the best design $b$ with $j_{\text {min }}=$ $\arg \min _{j \in\{1,2, \ldots, m\}} \mathcal{R}_{b j, l_{j} r_{l_{j}}}$. That is, we allocate $\Delta$ to the scenario in $\mathscr{S}_{1}$ with the minimal $\mathcal{R}_{b j, l_{j} r_{l_{j}}}$ value. This is because in $\mathscr{S}_{1}$, scenario $j_{\text {min }}$ and scenario $j_{\max }$ with $j_{\text {max }}=\arg \max _{j \in\{1,2, \ldots, m\}} \mathcal{R}_{b j, l_{j} r_{l_{j}}}$ correspond to the largest violation of the optimality condition (10). According to (15), we can allocate additional replications to scenario $j_{\min }$ to alleviate or eliminate this violation. Similarly, if $\mathcal{A}_{1} \geq \mathcal{A}_{2}$, we allocate the incremental budget $\Delta$ to the worst-case scenario of non-best design $l_{\text {min }}$ with $l_{\text {min }}=\arg \min _{l \in\{1,2, \ldots, k\}, l \neq b} \mathcal{R}_{b j_{l}, l r_{l}}$. That is, we allocate $\Delta$ to the scenario in $\mathscr{S}_{2}$ with the minimal $\mathcal{R}_{b j_{l}, l r_{l}}$ value.

Note that the above conclusions are based on the fact that the sets $\mathscr{S}_{1}$ and $\mathscr{S}_{2}$ are known. In practice, they need to be estimated. Therefore, we still need to simulate all scenarios under consideration, although the optimality conditions (9), (10) and (11) suggest to concentrate the simulation budget on scenarios in $\mathscr{S}_{1}$ and $\mathscr{S}_{2}$. At the beginning, we provide the same number of simulation replications for all the scenarios and estimate $\mathscr{S}_{1}$ and $\mathscr{S}_{2}$. In the following iterations, we allocate the simulation budget using the method described above. We call it R-OCBA (robust OCBA) procedure.

\section{R-OCBA Procedure}

1. Specify the total simulation budget $n$, the initial simulation replication number $n_{0}$ and the incremental budget $\Delta_{0}$. Iteration counter $\kappa \leftarrow 1$. Perform 
$n_{0}$ replications to all the scenarios. $n_{i, j}^{\kappa}=n_{0}$ for all $i=1,2, \ldots, k$ and $j=1,2, \ldots, m$.

2. Calculate sample mean $\bar{X}_{i, j}$ and sample variance $S_{i, j}^{2}, i=1,2, \ldots, k$ and $j=1,2, \ldots, m$.

3. If $\sum_{i=1}^{k} \sum_{j=1}^{m} n_{i, j}^{\kappa}=n$, go to Step 4. Otherwise, repeat

a. Calculate the estimates $\hat{\mathscr{S}}_{1}, \hat{\mathscr{S}}_{2}, \hat{\mathcal{A}}_{1}, \hat{\mathcal{A}}_{2}, \hat{b}$, $\hat{j}_{\text {min }}, \hat{l}_{\text {min }}$ and $\hat{r}_{\hat{l}_{\text {min }}}$ for $\mathscr{S}_{1}, \mathscr{S}_{2}, \mathcal{A}_{1}, \mathcal{A}_{2}, b$, $j_{\text {min }}, l_{\text {min }}$ and $r_{l_{\text {min }}}$ using $\bar{X}_{i, j}$ and $S_{i, j}^{2}$.

b. Provide an incremental simulation budget $\Delta=\min \left\{\Delta_{0}, n-\sum_{i=1}^{k} \sum_{j=1}^{m} n_{i, j}^{\kappa}\right\}$. If $\hat{\mathcal{A}}_{1}<\hat{\mathcal{A}}_{2}$, allocate $\Delta$ to scenario $\hat{j}_{\text {min }}$ of de$\operatorname{sign} \hat{b}, n_{\hat{b}, \hat{j}_{\text {min }}}^{\kappa+1}=n_{\hat{b}, \hat{j}_{\text {min }}}^{\kappa}+\Delta$; otherwise, allocate $\Delta$ to the scenario $\hat{r}_{\hat{l}_{\text {min }}}$ of design $\hat{l}_{\text {min }}$, $n_{\hat{l}_{\min }, \hat{r}_{\hat{l}_{\text {min }}}}^{\kappa+1}=n_{\hat{l}_{\text {min }}, \hat{r}_{\hat{l}_{\text {min }}}}^{\kappa}+\Delta$.

c. Update $\bar{X}_{i, j}$ and $S_{i, j}^{2}$ of the scenario receiving additional replications.

d. $\kappa \leftarrow \kappa+1$.

4. Select design $\hat{b}$ as the best design.

Remark 1 Note that $\bar{X}_{i, j}$ and $S_{i, j}^{2}$ are consistent estimators of $\mu_{i, j}$ and $\sigma_{i, j}^{2}$. During the budget allocation process, it is possible that the estimated best design $\hat{b} \neq b$ due to the estimation errors. However, as the allocation proceeds, more replications will be allocated to the $m$ scenarios of design $\hat{b}$ and the estimated worst-case scenarios of the rest designs. Then, the worst-case scenario of design $\hat{b}$ will be correctly observed. Since all the $m$ scenarios of design $b$ are superior to the worst-case scenario of design $\hat{b}$, the estimated worst-case scenario of design $b$ will become better than that of design $\hat{b}$. That is, as the allocation proceeds, $\hat{b}$ will converge to $b$.

\section{Numerical Experiments}

In this section, we test the proposed R-OCBA procedure by comparing it with existing approaches. The following three selection approaches are used for comparison:

- Equal Allocation (EA): The total simulation budget is equally allocated to each scenario, so that all the scenarios are simulated equally often. The performance of equal allocation serves as a benchmark for comparison.

- Proportional-to-Variance (PTV) Allocation: The twostage PTV procedure has been commonly used in the simulation literature (Rinott, 1978). In the first stage, all scenarios are simulated for the same number of replications. The number of additional simulation replications for each scenario in the second stage is proportional to their sample variances. In this test, we implement PTV sequentially for a fair comparison.

- Robust Selection-of-the-Best (RSB) Allocation: RSB is a two-layer procedure, either two-stage or fully se- quential, to select the best simulated design under input uncertainty. It adopts the IZ formulation. In the first layer, RSB identifies the worst-case scenario of each design and in the second layer, it selects the best design. To the best of our knowledge, RSB is the only selection procedure in the literature with the same purpose of the proposed R-OCBA procedure. In this test, we will use the sequential version of RSB.

To compare EA, PTV, RSB and R-OCBA, we use four typical selection problems:

- Example 1: 100 scenarios and constant variances. This example has $k=20$ designs and each design has $m=$ 5 scenarios. Simulation samples of each scenario are normally distributed with $\mu_{i, j}=i+j-1$ and $\sigma_{i, j}^{2}=8^{2}$, $i=1,2, \ldots, 20$ and $j=1,2, \ldots, 5$. The best design is design 1.

- Example 2: 100 scenarios and increasing variances. This example has $k=20$ designs and each design has $m=5$ scenarios. Simulation samples of each scenario are normally distributed with $\mu_{i, j}=i+j-1$ and $\sigma_{i, j}^{2}=(6.5+0.5 j)^{2}, i=1,2, \ldots, 20$ and $j=1,2, \ldots, 5$. The best design is design 1 .

- Example 3: 100 scenarios and decreasing variances. This example has $k=20$ designs and each design has $m=5$ scenarios. Simulation samples of each scenario are normally distributed with $\mu_{i, j}=i+j-1$ and $\sigma_{i, j}^{2}=(9.5-0.5 j)^{2}, i=1,2, \ldots, 20$ and $j=1,2, \ldots, 5$. The best design is design 1 .

- Example 4: 32-scenario emergency room staffing problem. This is a test problem in Simulation Optimization Library (http://www.simopt.org/). Each design is in form of $\left(x^{1}, x^{2}, x^{3}, x^{4}, x^{5}\right)$, where $x^{1}, x^{2}, x^{3}, x^{4}$ and $x^{5}$ represent the number of receptionists, doctors, lab technicians, treatment nurses and emergency room nurses respectively. We consider 8 designs: $(1,3,2,1,7),(1,3,2,1,9),(1,3,2,2,6)$, $(1,3,2,2,7), \quad(1,3,2,2,8), \quad(1,3,2,3,7),(1,3,2,5,5)$ and $(1,3,2,6,7)$. A scenario corresponds to a service time distribution in the examination room. We consider 4 scenarios for each design: exponential distributions with a mean parameter of $12,13,14$ and 15 respectively. For each design, the performance of a scenario is measured by the mean waiting time of critical patients. The best design is $(1,3,2,1,9)$, which is determined by comparing the sample average (200 replications) of all the scenarios.

Among the four methods, RSB must be implemented differently. It keeps increasing the simulation budget until PCS is guaranteed at the pre-specified level $P_{0}$, while the other three methods should be performed with a fixed simulation budget. In order to compare the four methods, we first run RSB and let $P_{0}$ take a number of different values. For each $P_{0}$ value, RSB is performed 4000 times, and the averaged total simulation budget and the averaged PCS are calculated. Then, we run EA, PTV 
and R-OCBA 4000 times and each time let the simulation budgets of the three methods be in the same range of the simulation budget of RSB. The averaged PCS values of EA, PTV and R-OCBA are then calculated and compared with those of RSB.

In RSB, $P_{0}$ is set as $0.2,0.25,0.3, \ldots, 0.8$ for all the four examples. As suggested by Fan et al. (2013), we let the error levels of the two layers $\beta_{1}=\beta_{2}=\left(1-P_{0}\right) /(k+$ $m-2)$. The IZ parameters $\delta_{1}=\delta_{2}=1$ for examples $1-3$ and $\delta_{1}=\delta_{2}=0.1$ for example 4 . The initial simulation replication number $n_{0}$ is 40 for all the scenarios. In EA, PTV and R-OCBA, $n_{0}=40$ and the incremental budget $\Delta_{0}=20$. We report the PCS values of the four methods in Fig. 1-4.

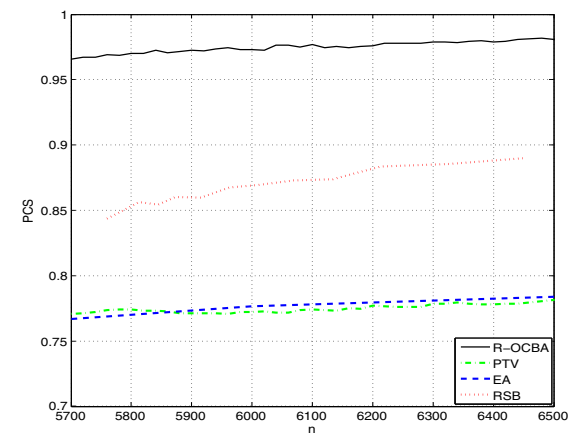

Fig. 1. PCS of the four methods for example 1.

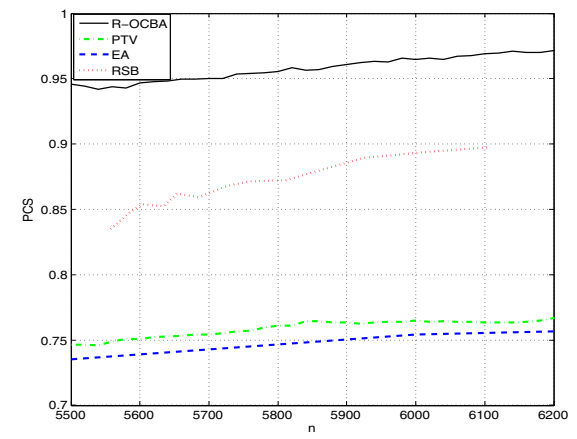

Fig. 2. PCS of the four methods for example 2 .

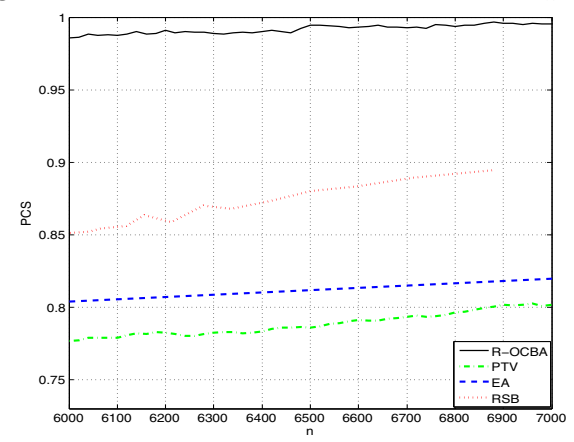

Fig. 3. PCS of the four methods for example 3.

It is observed that the proposed R-OCBA procedure is significantly superior to the methods in comparison. It leads to higher PCS with different simulation budgets

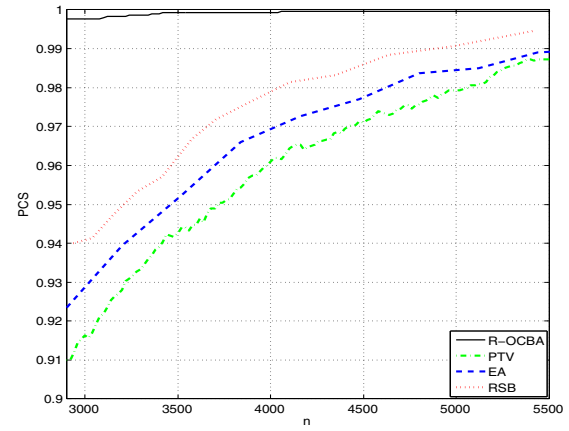

Fig. 4. PCS of the four methods for example 4.

for all the tested examples. As discussed in Section 3.1, R-OCBA is efficient primarily because it concentrates the simulation budget on scenarios in sets $\mathscr{S}_{1}$ and $\mathscr{S}_{2}$. Then, if $\mathscr{S}_{1}$ and $\mathscr{S}_{2}$ can be accurately estimated in the initial stage, R-OCBA substantially reduces the scale of the original problem (from $\mathrm{km}$ scenarios to $k+m-1$ scenarios), and thus can solve the selection problem with higher efficiency.

The performance of RSB is second to R-OCBA in the compared methods. RSB is based on IZ parameters and thus might allocate more than enough simulation replications for some scenarios. EA and PTV perform the worst. This is not surprising because these two methods do not appear to have any efficient mechanism for selecting the best design.

\section{Conclusions}

In this study, we considered R\&S problems with input uncertainty. The designs under consideration are assumed to have a finite number of scenarios in terms of input distribution, and the objective is to allocate the simulation budget to all the scenarios to efficiently select the design with the best worst-case scenario. We developed an approximation for the probability of correct selection, and then derived an asymptotic optimal solution for the selection problem. A corresponding sequential selection procedure, called R-OCBA, is designed for implementation purposes. A series of numerical experiments indicate that R-OCBA is considerably more efficient than the existing methods in the literature.

\section{References}

Barton, R., Nelson, B., \& Xie, W. (2014). Quantifying input uncertainty via simulation confidence intervals. INFORMS Journal on Computing, 26, 74-87.

Ben-Tal, A., den Hertog, D., Waegenaere, A. D., Melenberg, B., \& Rennen, G. (2013). Robust solutions of optimization problems affected by uncertain probabilities. Management Science, 59, 341-357. 
Branke, J., Chick, S. E., \& Schmidt, C. (2007). Selecting a selection procedure. Management Science, 53, 1916-1932.

Chen, C. H., Lin, J., Yücesan, E., \& Chick, S. E. (2000). Simulation budget allocation for further enhancing the efficiency of ordinal optimization. Discrete Event Dynamic Systems, 10, 251-270.

Chen, W., Gao, S., Chen, C.-H., \& Shi, L. (2014). An optimal sample allocation strategy for partition-based random search. IEEE Transactions on Automation Science and Engineering, 11, 177-186.

Corlu, C. G., \& Biller, B. (2013). A subset selection procedure under input parameter uncertainty. In Proceedings of the 2013 Winter Simulation Conference (pp. 463-473)

Delage, E., \& Ye, Y. (2010). Distributionally robust optimization under moment uncertainty with application to data-driven problems. Operations Research, 58, 595-612.

Fan, W., Hong, L. J., \& Zhang, X. (2013). Robust selection of the best. In Proceedings of the 2013 Winter Simulation Conference (pp. 868-876).

Gao, S., \& Chen, W. (2015). Efficient subset selection for the expected opportunity cost. Automatica, 59, 19-26.

Gao, S., \& Chen, W. (2016a). Efficient feasibility determination with multiple performance measure constraints. IEEE Transactions on Automatic Control, . In press.

Gao, S., \& Chen, W. (2016b). A new budget allocation framework for selecting top simulated designs. IIE Transaction, . In press.

Gao, S., \& Shi, L. (2015). Selecting the best simulated design with the expected opportunity cost bound. IEEE Transactions on Automatic Control, 60, 27852790.

Gao, S., Xiao, H., Zhou, E., \& Chen, W. (2016). Optimal computing budget allocation with input uncertainty. In Proceedings of the 2016 Winter Simulation Conference (pp. 839-846).

Ghaoui, L. E., Oks, M., \& Oustry, F. (2003). Worstcase value-at-risk and robust portfolio optimization: a conic programming approach. Operations Research, $51,543-556$.

Glynn, P., \& Juneja, S. (2004). A large deviations perspective on ordinal optimization. In Proceedings of the 2004 Winter Simulation Conference (pp. 577-585).

Gülpınar, N., \& Rustem, B. (2007). Worst-case robust decisions for multi-period mean-variance portfolio optimization. European Journal of Operational Research, 183, 981-1000.

Kim, S. H., \& Nelson, B. L. (2001). A fully sequential procedure for indifference-zone selection in simulation. ACM Transactions on Modeling and Computer Simulation, 11, 251-273.

Rinott, Y. (1978). On two-stage selection procedures and related probability inequalities. Communications in Statistics, A7, 799-811.

Song, E., Nelson, B., \& Pegden, D. (2014). Advanced tutorial: input uncertainty quantification. In Proceedings of the 2014 Winter Simulation Conference (pp. 162-176). 